\title{
Erratum to: Smart pathological brain detection system by predator-prey particle swarm optimization and single-hidden layer neural-network
}

\author{
Hainan Wang ${ }^{1} \cdot$ Yiding $\mathrm{Lv}^{1} \cdot$ Hong Chen ${ }^{2} \cdot$ Yujie $\mathrm{Li}^{3} \cdot$ \\ Yin Zhang ${ }^{4} \cdot$ Zhihai Lu $^{1}$
}

(C) Springer Science+Business Media New York 2017

\section{Erratum to: Multimed Tools Appl \\ DOI 10.1007/s11042-016-4242-0}

In the original publication, the Fig. 3 was incorrectly presented. The white background of " 44 " in Fig. $3 b$ should be removed. This figure was corrected in the original.

The online version of the original article can be found at http://dx.doi.org/10.1007/s11042-016-4242-0

\section{Yujie Li}

liyujie@yzu.edu.cn

$\triangle$ Yin Zhang

yin.zhang.cn@ieee.org

$\triangle$ Zhihai Lu

luzhihai@njnu.edu.cn

1 School of Computer Science and Technology \& School of Education Science, Nanjing Normal University, Nanjing, Jiangsu 210023, China

2 Department of Neurology, First Affiliated Hospital of Nanjing Medical University, Nanjing 210029, China

3 School of Information Engineering, Yangzhou University, Yangzhou, China

4 School of Information and Safety Engineering, Zhongnan University of Economics and Law, Wuhan, Hubei 430073, China 